Voix et Images

\title{
L'espace mythique dans Kamouraska de M. Bernadette Velloso Porto
}

\section{Élène Cliche}

Volume 12, numéro 1 (34), automne 1986

Québec-Amérique latine

URI : https://id.erudit.org/iderudit/200609ar

DOI : https://doi.org/10.7202/200609ar

Aller au sommaire du numéro

Éditeur(s)

Université du Québec à Montréal

ISSN

0318-9201 (imprimé)

1705-933X (numérique)

Découvrir la revue

Citer cet article

Cliche, É. (1986). L'espace mythique dans Kamouraska de M. Bernadette Velloso Porto. Voix et Images, 12(1), 106-108. https://doi.org/10.7202/200609ar d'utilisation que vous pouvez consulter en ligne.

https://apropos.erudit.org/fr/usagers/politique-dutilisation/ 


\title{
Essai \\ L'Espace mythique dans Kamouraska de M. Bernadette Velloso Porto
}

\author{
par Êlène Cliche, Université du Québec à Montréal
}

Il importe de souligner l'existence et l'intérêt d'une thèse de doctorat brésilienne ${ }^{1}$ sur Kamouraska d'Anne Hébert, écrite en portugais par Maria Bernadette Thereza Velloso Porto, et soutenue en 1983 à la Faculté des Lettres de l'Université Fédérale de Rio de Janeiro, au Département de Langue et Littérature française.

Dans sa présentation initiale de l'œuvre, M. B. Velloso Porto signale que chez Anne Hébert, la parole poétique assume la fonction d'un acte sacré à caractère épiphanique. Poésie et révélation se confondent à tel point que le monde antérieur à la manifestation de la parole magique et transformatrice n'est plus qu'un espace de ténèbres et d'incommunicabilité. C'est à partir de cette reconstitution de la vie dramatique de la parole que l'auteure de la thèse opère d'emblée un rapprochement pertinent entre Anne Hébert et la célèbre écrivaine brésilienne Clarice Lispector qui considère également l'écriture comme une chose sacrée où les infidèles n'ont pas d'entrée ${ }^{2}$.

L'auteure cherche surtout à mettre en valeur la complexité de Kamouraska, malgré son apparence de simplicité. Ses présupposés théoriques, bien expliqués dans le premier chapitre, ne prétendent pas conférer au roman étudié un sens ultime et totalisant, mais plutôt à montrer comment, grâce à diverses entrées de lecture, nous sommes amenés (es) à reconnaître l'interrelation entre des niveaux distincts ouvrant le texte vers une dimension mythique, caractérisée essentiellement par un principe "d'indistinction" des éléments.

Ainsi, les trois grandes parties de la thèse, qui constituent respectivement des chapitres, correspondent à trois plans différents d'analyse. Le premier est la stratégie du récit, A estratégia da narrativa où sont examinées successivement les catégories voix, mode, temps, d'après Genette dans Figures III. En outre, ceci permet l'étude de la mise en abyme dans Kamouraska, du circuit mobile des pronoms personnels, de la fonction phatique du discours illustrée par des exemples pertinents. Sur le mode du récit, il y a des remarques subtiles sur le non-dit textuel, dans le jeu des sous-conversations selon la définition de Sarraute dans l'Êre du soupçon. Grâce à des incursions multiples, l'auteure prouve - en rapport avec le principe d'indistinction - qu'il n'existe pas de frontières entre les diverses voix, c'est-à-dire que la parole, à valeur d'écho, peut être assumée par des personnages variés.

Aussi, la complexité temporelle, où toute idée de linéarité est exclue, amène à considérer l'espace textuel d'un point de vue psychanalytique, comme superposition de temps, lieux et images. Ceci, grâce à la condensation des éléments et à la capacité du récit à répéter des segments temporels par exemple, que l'analyse de la fréquence permet de vérifier. La mémoire du personnage 
central Élisabeth, femme-sorcière, "feiticeira», dont les pouvoirs magiques lui permettent de se déplacer au-delà des contraintes spatio-temporelles, y est pour quelque chose dans l'organisation de cette autre logique du récit s'engendrant à partir de pièces de souvenirs ("cacos de lembranças" p. 71), de fragments de rêves, d'expectatives. Or, les faits d'ubiquité, de réversibilité et d'itération observés au niveau du récit contribuent à établir un temps et un éspace particuliers. annonçant déjà la place du mythe dans le roman.

Après diverses considérations narratologiques, nous passons à un deuxième plan, celui des jeux et du sacré, Do espaço, dos jogos ao sagrado, espace où se marque encore l'indistinction, cette fois des doubles, des partenaires et des rivaux. L'auteure s'interroge sur les liens entre la transgression, les excès violents et le jeu érotique et mortel, celui-ci étant assimilé au sacrifice tragique. Ce chapitre nous apparaît comme étant le plus riche et le plus stimulant, bien qu'il serait malvenu de notre part d'intervenir, de quelque façon, sous l'instance du «juge", la thèse ayant déjà subi l'épreuve de la sanction. Cependant, alors que l'analyse narratologique de Kamouraska nous semble déjà plus familière ou plus commune, quoiqu'elle soit ici fine et originale, s'attachant au plus près du signifiant (pourtant en langue étrangère pour celle qui la fait), cette deuxième investigation, où la lecture cherche à vérifier jusqu'à quel point le domaine du jeu s'articule et se confond avec le sacré, est du plus grand intérêt.

Ici, la classification des quatre catégories ludiques, alea (hasard), agon (compétition), ilinx (vertige), mimicry (simulacre), établies par Roger Caillois dans les Jeux et les hommes, Gallimard, 1958, s'avère très efficace, car elle est bien utilisée pour mettre en valeur ce qui, sous différents angles, se joue précisément dans le texte. Celui-ci n'est pas écrasé par ce qui pourrait être une "grille» (c'est avec raison que ce mot nous fait horreur) asphyxiante. À titre d'exemple, l'étude des manifestations agonistiques et des conflits entre les personnages dans l'interaction textuelle est des plus brillantes. En se basant sur le modèle de la chasse et du potlatch primitif (travaux de Mauss), l'auteure met en scène les isotopies de l'érotisme et du sacrifice de la chair, de la part maudite (Bataille) où la notion d'échange symbolique est à la base du crime envisagé comme acte réversible et rituel anthropophage. L'éclairage que donne Bataille sur la dépense dans le fonctionnement du potlatch permet une lecture intense de la relation agonistique entre Antoine et Élisabeth dans le roman d'Anne Hébert.

À la lumière également des analogies établies par René Girard entre la violence contagieuse et le sacré, M.B. Velloso Porto cherche à voir si le jeu violent et érotique conduit à la domination du sacré. Envisagés par rapport à la notion de "crise sacrificielle», les personnages sont des victimes du désir mimétique, où chacun imite le désir de destruction de l'autre. Le processus multiplie les effets de miroir entre les adversaires définis comme des doubles. L'auteure pousse encore plus loin son analyse par le biais du pharmakon (cf. "La pharmacie de Platon» dans la Dissémination de Derrida), surtout en relation avec la fonction exercée par George Nelson dans le roman. Toujours dans l'optique derridienne, le pouvoir de substitution du supplément est pris 
en considération, l'homme tenant lieu ici d'excédent dans un jeu illimité, tel un «joker», carte neutre à la disposition de la maîtresse du jeu érotique.

La rencontre fructueuse de ces théories forme une systématique cohérente pour l'analyse du rite érotique et sacrificiel, avec l'ambivalence des doubles où s'accomplit un échange organique entre vie et mort, perte et renaissance dans une circulation mythique sans fin. L'étude des autres catégories ludiques permet également des greffes qui fertilisent la problématique élaborée plus haut.

Le troisième et dernier plan de l'analyse renvoic aux deux précédents, mais ce sont les configurations de l'imaginaire qui seront ici mises en relief. Nous retrouvons le principe d'indistinction, en l'occurrence une confusion des éléments dans les deux régimes de l'imaginaire, diurne et nocturne, selon la typologie bien connue de Gilbert Durand, dont l'auteure se sert pour son évaluation. Les «structures anthropologiques" relevées par Durand sont utiles comme instrument heuristique, mais sa théorie est malgré tout mise en échec par le travail textuel du supplément ou de l'effacement des différences entre les paires antithétiques dans Kamouraska. Comme quoi le texte littéraire, par sa dynamique, remet toujours en question, heureusement, la fixité du sens des symboles. Par rapport à ceux-ci, une étude minutieuse des images récurrentes est faite: la femme fatale ambivalente, inquiétante et protectrice, donnant la vie et la mort. La lune et le nombre trois dans le roman, le bestiaire et le cercle tragique, comportant aussi des valeurs contradictoires. Celui-ci est relié à l'itératif au niveau du récit et à l'échange symbolique entre les adversaires à un deuxième niveau. L'analyse insiste cependant sur la maîtrise du rythme circulaire par la narratrice, incarnation de la fileuse mythique qui permet en définitive la victoire de l'imagination et de la mémoire sur la putréfaction de la mort. Grâce à ses dons magiques et à la valeur performative de sa parole, Élisabeth préfigure Sœur Julie de la Trinité dans les Enfants du Sabbat.

À ce point final, l'étude du récit, de l'espace ludique et symbolique de Kamouraska nous amène à penser qu'il y a dans la lecture productive de $\mathbf{M}$. Bernadette Velloso Porto un travail du désir qui tisse lui-même la circularité du texte. D'une manière éminemment positive, ceci rejoint la «clairvoyance folle" d'Élisabeth dans le vertige du roman.

1. La thèse intitulée $\mathbf{O}$ espaço mitico em Kamouraska (215 pages) peut être consultée au Centre de Documentation du Département d'Études littéraires de l'Université du Québec à Montréal. Actuellement, elle n'est pas traduite en français. Cependant, toutes les citations de Kamouraska sont extraites de l'édition originale, publiée à Paris, Seuil, 1970.

2. "Croisa sagrada onde os infiéis nao têm entrada " in C. Lispector, Um sopro de vida, 1978, cité p. 13. La thèse de M.B. Velloso Porto est parsemée de points d'appui citationnels provenant de l'oeuvre de Clarice Lispector; plusieurs exergues en sont la manifestation. Cet enclenchement dialogique a donné lieu également à une remarquable étude "comparative» écrite en français par M.B. Velloso Porto, où celle-ci fait émerger des points de contact entre Anne Hébert et Clarice Lispector. cf. La via crucis du corps: Lecture intertextuelle d'Anne Hébert et de Clarice Lispector dans les Cahiers du CEF, Cercle d'Études francophones, Université Fédérale Fluminense, Niteroi, Rio de Janeiro. Brésil, no 1 intitulé «Le Québec vu du Brésil», Mars 1985, p. 53-82. 